\title{
LINEARLY CONTINUOUS FUNCTIONS ${ }^{1}$
}

\author{
CASPER GOFFMAN AND C. J. NEUGEBAUER
}

In [1], G. Tolstoff showed that linearly continuous real functions of two real variables are determined by their values on dense sets. Tolstoff's proof is complicated and uses analytic sets. Moreover, his argument does not apply to higher dimensions. In this note we give a short and elementary proof of Tolstoff's theorem for the $n$-dimensional case.

Let $E_{1}$ be the real line, and let $R_{k}=E_{1}, k=1,2, \cdots$. Let $E_{n}=R_{1} \times \cdots \times R_{n}, n=1,2, \cdots$, be the euclidean $n$-space.

Definition. A function $f: E_{n} \rightarrow E_{1}$ is said to be linearly continuous if for every $\left(x_{1}^{0}, \cdots, x_{n}^{0}\right) \in E_{n}$ and $i=1, \cdots, n$ the function $f\left(x_{1}^{0}, \cdots, x_{i-1}^{0}, \cdot, x_{i+1}^{0}, \cdots, x_{n}^{0}\right): R_{i} \rightarrow E_{1}$ is continuous at $x_{i}^{0}$.

THEOREM 1. If $f$ and $g$ are linearly continuous on $E_{n}$ and agree on a dense set, then they are identical. ${ }^{2}$

Proof. It suffices to show that if there is a $p \in E_{n}$ for which $f(p)$ $>k>0$, then there is an open set $G \subset E_{n}$ such that $f\left(q_{0}\right) \geqq k$ for every $q_{0} \in G$.

This statement is evidently true for $n=1$. We proceed by induction, and we suppose the statement is true for $n-1$. Let $f: E_{n} \rightarrow E_{1}$ be linearly continuous and $f(p)>k>0$ at $p=\left(x_{1}^{0}, \cdots, x_{n}^{0}\right)$. There is an open interval $I \subset R_{n}$ such that $f\left(x_{1}^{0}, \cdots, x_{n-1}^{0}, x_{n}\right)>k, x_{n} \in I$. By the induction hypothesis, for every $x_{n} \in I$ there is an open rational cube $K\left(x_{n}\right)$ such that $f\left(x_{1}, \cdots, x_{n-1}, x_{n}\right) \geqq k$ for every $\left(x_{1}, \cdots, x_{n-1}\right)$ $\in K\left(x_{n}\right)$. Since $I$ is of the second category, there is an open interval $\exists \subset I$, a dense set $S \subset$ J, and a fixed open cube $K$ such that $f\left(x_{1}, \cdots, x_{n}\right)$ $\geqq k$ for all $\left(x_{1}, \cdots, x_{n-1}\right) \in K$ and $x_{n} \in S$. Since $f$ is continuous in $x_{n}$ for every $\left(x_{1}, \cdots, x_{n-1}\right)$, it follows that $f\left(x_{1}, \cdots, x_{n}\right) \geqq k$ on the open cube $K \times \mathfrak{J C} E_{n}$.

We note that the same reasoning yields the following result.

TheOREM $1^{\prime}$. If $X_{1}, \cdots, X_{n}$ are second countable Baire spaces, $Y$ is a Baire space, $Z$ is a first countable topological space, $M$ is a regular Hausdorff space, and $f$, g are separately continuous on $Y \times Z \times X_{1} \times \cdots$ $\times X_{n}$ to $M$, i.e., continuous in each variable separately, then, if $f$ and $g$ agree on a dense set, they are identical.

In conclusion, we observe that the condition that the spaces are

Received by the editors July $15,1961$.

${ }^{1}$ Supported by National Science Foundation Grant NSF G-18920.

$2 \mathrm{~A}$ short proof can also be given using quasi-continuity. 
Baire spaces cannot be removed. If $Q$ is the space of rationals in $E_{1}$ with the relative topology, there is a separately continuous $f: Q \times Q$ $\rightarrow E_{1}$ which is zero on a dense subset of $Q \times Q$ but not identically zero.

\section{REFERENCE}

1. G. Tolstoff, On partial derivatives, Amer. Math. Soc. Transl. No. 69; Izv. Akad. Nauk SSSR Ser. Mat. vol. 13 (1949) pp. 425-449.

PuRdue University

\section{COMPLETE SEQUENCES OF FUNCTIONS ${ }^{1}$}

\section{CASPER GOFFMAN}

Although the result of this note is implicitly contained in the work of A. A. Talalyan [2] and could also have been a corollary to the theorem in [1], it seems to be of sufficient interest to merit explicit treatment.

It is known (see [1]) that if $\left\{f_{1}, f_{2}, \cdots, f_{n}, \cdots\right\}$ is a sequence of measurable functions which is complete in the space $M$ of measurable functions (i.e., every measurable $f$ is the limit in measure of a sequence of finite linear combinations of $\left.\left\{f_{1}, f_{2}, \cdots, f_{n}, \cdots\right\}\right)$ then $\left\{f_{2}, f_{3}, \cdots, f_{n}, \cdots\right\}$ is also complete in $M$.

Let $X$ be a separable Banach space of measurable functions on $[a, b]$ such that for every measurable $G \subset[a, b]$, with $m(G)>0$, the set $X_{G}$ of restrictions of the functions in $X$ to $G$ is a Banach space and

(a) If $\left\{g_{n}\right\}$ converges to $g$ in $X$ then $\left\{g_{n}\right\}$ converges to $g$ in $X_{G}$,

(b) The set of bounded measurable functions is a dense subset of $X$; hence, of $X_{G}$, for every $G$,

(c) For every $G$, uniform convergence on $G$ implies convergence in $X_{G}$ and convergence in $X_{G}$ implies convergence in measure on $G$.

THEOREM. If $\left\{f_{1}, f_{2}, \cdots, f_{n}, \cdots\right\}$ is complete in $X$ and $\epsilon>0$, there is a measurable $G \subset[a, b]$, with $m(G)>(b-a)-\epsilon$, such that $\left\{f_{2}, f_{3}, \cdots, f_{n}, \cdots\right\}$ is complete in $X_{G}$.

Proof. Let $\left\{g_{1}, g_{2}, \cdots, g_{n}, \cdots\right\}$ be dense in $X$. Since $\left\{f_{1}, f_{2}, \cdots, f_{n}, \cdots\right\}$ is complete in $X$, it follows from (b), (c) and

Received by the editors July 18, 1961 .

1 Supported by National Science Foundation Grant NSF-G18920. 